\title{
Coagulopathy after hemorrhagic traumatic brain injury, an observational study of the incidence and prognosis
}

\author{
Jort A. N. van Gent ${ }^{1,2,3}$ - Thomas A. van Essen ${ }^{1,2,3}$ - Mettine H. A. Bos ${ }^{4}$ - Suzanne C. Cannegieter ${ }^{4,5}$. \\ Jeroen T. J. M. van Dijck ${ }^{1,2,3}$. Wilco C. Peul ${ }^{1,2,3}$
}

Received: 16 August 2019 / Accepted: 14 October 2019 / Published online: 18 November 2019

(C) The Author(s) 2019

\begin{abstract}
Background Traumatic brain injury is associated with high rates of mortality and morbidity. Trauma patients with a coagulopathy have a 10-fold increased mortality risk compared to patients without a coagulopathy. The aim of this study was to identify the incidence of coagulopathy and relate early coagulopathy to clinical outcome in patients with traumatic intracranial hemorrhages. Methods Between September 2015 and December 2016, 108 consecutive cranial trauma patients with traumatic intracranial hemorrhages were included in this study. To assess the relationship between patients with a coagulopathy and outcome, a chisquared test was performed.

Results A total of 29 out of the 108 patients (27\%) with a traumatic intracranial hemorrhage developed a coagulopathy within $72 \mathrm{~h}$ after admission. Overall, a total of 22 patients $(20 \%)$ died after admission of which ten were coagulopathic at emergency department presentation. Early coagulopathy in patients with traumatic brain injury is associated with progression of hemorrhagic injury (odds ratio 2.4 (95\% confidence interval 0.8-8.0)), surgical intervention (odds ratio 2.8 (95\% confidence interval $0.87-$ 9.35)), and increased in-hospital mortality (odds ratio 23.06 (95\% confidence interval 5.5-95.9)).

Conclusion Patients who sustained a traumatic intracranial hemorrhage remained at risk for developing a coagulopathy until $72 \mathrm{~h}$ after trauma. Patients who developed a coagulopathy had a worse clinical outcome than patients who did not develop a coagulopathy.
\end{abstract}

Keywords Traumatic brain injury $\cdot$ Coagulopathy $\cdot$ Progression of hemorrhagic injury $\cdot$ Time course $\cdot$ Mortality

Jort A. N. van Gent and Thomas A. van Essen contributed equally to this work.

This article is part of the Topical Collection on Brain trauma

Jort A. N. van Gent

J.A.N.van_Gent@lumc.nl

Thomas A. van Essen

T.A.van_Essen@lumc.nl

Mettine H. A. Bos

M.H.A.Bos@lumc.nl

Suzanne C. Cannegieter

S.C.Cannegieter@lumc.nl

Jeroen T. J. M. van Dijck

J.T.J.M.van_Dijck@lumc.nl
Wilco C. Peul

W.C.Peul@lumc.nl

1 Department of Neurosurgery, Leiden University Medical Center, University Neurosurgical Center Holland (UNCH), Albinusdreef 2, 2333 ZA Leiden, The Netherlands

2 HAGA Teaching Hospital, The Hague, The Netherlands

3 Haaglanden Medical Center, The Hague, The Netherlands

4 Division of Thrombosis and Hemostasis, Leiden University Medical Center, Leiden, The Netherlands

5 Department of Clinical Epidemiology, Leiden University Medical Center, Leiden, The Netherlands 


\section{Introduction}

Traumatic brain injury (TBI) is a major cause of mortality and disability globally, and unfortunately, mortality and neurological morbidity has not decreased substantially over the last 30 years $[9,12,28]$. One of the most significant parameters that influences patient outcome is the presence of a traumatic intracranial hemorrhage $(\mathrm{t}$ ICH). Patients sustaining moderate and severe TBI (i.e., a Glasgow Coma Scale (GCS) between 3 and 12) often have intracranial bleeding such as an acute subdural hemorrhage $(\mathrm{ASDH})$, an epidural hemorrhage (EDH), a cerebral contusion (CC), a subarachnoid hemorrhage (SAH) or a combination of them, which can be accompanied by secondary injury. This secondary injury may consist of brain swelling and progression of hemorrhagic injury (PHI) which, ultimately, could form a risk of herniation $[12,23]$. For the focal lesions ASDH, EDH, and/ or contusions, surgical evacuation is the cornerstone of treatment. Creating optimal conditions for surgical treatment and prevention of PHI can have a major impact on mortality and morbidity [4]. One of these conditions is a proper hemostasis. Unfortunately, after TBI, hemostasis is often derailed, leading to a hypocoagulopathic andless often-a hypercoagulopathic state [17]. Multiple studies have found that one third of all patients who sustained a TBI developed a coagulopathy due to trauma [8]. Other studies related such a trauma-induced coagulopathy with PHI and found it to be a powerful independent risk factor for mortality $[1,7,11,25]$. Patients with a coagulopathy after TBI have a 10 -fold increased mortality risk in addition to a 30 -fold higher risk of unfavorable outcome $[8,17]$.

While the pathophysiological mechanisms are largely unknown, one of the most likely hypothesis is that after a TBI, the naturally occurring abundance of tissue factor (TF) in the microvessels of the brain is massively released into the bloodstream [13]. This triggers the coagulation cascade via the external coagulation pathway leading to consumptive insufficiency of coagulation factors [14]. Another leading (additional) hypothesis is that a combination between shock and hypoperfusion results in activation of protein $C[5,21]$, which promotes fibrinolysis through inactivation of plasminogen activator inhibitor-1 [27]. These hypotheses are mainly theorized and based on scarce empirical evidence.

Thus, there is a lack of insight into TBI-associated coagulopathies that sharply contrasts with their potential clinical consequences. First and foremost, it is unknown whether or not the coagulopathy is associated with intracranial hemorrhage after trauma. This multicenter cohort study aims to describe the incidence and time course of coagulopathy in patients with t-ICH. Additionally, the relation between TBIassociated coagulopathy and outcome will be explored.

\section{Material and methods}

\section{Study population}

Consecutive adult patients with TBI after isolated blunt head trauma between September 2015 and December 2016 were identified from the emergency department (ED) registry in three cooperating level 1 trauma centers within the University Neurosurgical Center Holland (Leiden University Medical Center, Haga Teaching Hospital, and Haaglanden Medical Centrum). In this recruitment period, the observational study CENTER-TBI was also active and did include the same patients as this study [24]. However, no data of the CENTER-TBI study was used. Patients were included when (1) they had suffered from TBI, (2) had presence of an intracranial hemorrhage (ASDH, EDH, SAH, CC) confirmed by the first posttrauma computed tomography (CT) scan, and (3) were primary presented to the participating hospital within $6 \mathrm{~h}$ after injury. Patients were excluded in case they (1) suffered from severe extracranial injuries (non-head Abbreviated Injury Score (AIS) > 3), (2) had no coagulation parameters available, (3) had a history of coagulation disorders, and (4) used anticoagulants. Medical Ethics Committee approval was granted (G16.087).

\section{Study parameters}

The following data were collected: demographic baseline data, GCS at ED presentation, extracranial AIS, medical history categorized by the American Society of Anesthesiologists Physical status Classification system (ASA) [6], surgeries performed on the patient, ICU admission, length of stay, and mortality. The use of anticoagulants or antifibrinolytics during hospital stay was not considered because this information was not readily available. Patients who died during hospital admission were categorized as follows: died within $24 \mathrm{~h}$ after hospital admission, died between 24 and $48 \mathrm{~h}$, died between 48 and $72 \mathrm{~h}$, or died within 72 and $96 \mathrm{~h}$ and died after $96 \mathrm{~h}$.

\section{Definition of coagulopathy}

Patients were categorized into a coagulopathy and a nocoagulopathy group, based on the coagulation profile drawn at presentation to the ED. Coagulopathy was defined as an international normalized ratio $($ INR) $>1.20$ and/or a platelet count below $150 \times 10^{3} / \mu 1$ and/or an activated partial thromboplastin time $($ APTT) $>34.5 \mathrm{~s}[2,31]$. To investigate the incidence of coagulation, blood drawn after $24,48,72$, and $96 \mathrm{~h}$ of admission was also included, if available. 


\section{Cranial computed tomography}

All evaluations of CT scans were extracted from the original report by the radiologist who was unaware of the study during the conduct of the report. PHI was defined as an increase in volume of the initial hemorrhage or development of new intracranial hemorrhages at sites distant from previous lesions, at the discretion of the attending radiologist. Patients who had no follow-up CT scan because they were clinically stable were classified as no PHI.

\section{Statistical analysis}

The mean and standard deviation (SD) or median and interquartile range (IQR) were calculated according to distribution and scale of measurement. To assess the relationship between patients who developed coagulopathy at ED presentation and mortality or PHI, a chi-squared test was performed with mortality and PHI as outcome variables. For these outcome variables, an odds ratio (OR) with a $95 \%$ confidence interval (CI) was calculated. All analyses were performed using the Statistical Package for Social Sciences (SPSS 25.0).

\section{Results}

\section{Subject recruitment}

From a total of 231 potential study patients with a t-ICH, 108 were included. Fifty-four patients were excluded because of anticoagulant usage, 29 patients were excluded because of secondary referral, and forty patients were excluded because of missing coagulation status at the participating hospital (Fig. 1).

\section{Baseline characteristics}

Median cohort age was 60 years (interquartile range (IQR) 31 ), and gender distribution was equal (Table 1). Based on the ASA physical status classification, only three patients (3\%) had a severe systemic disease. Most patients were diagnosed with an $\mathrm{ASDH}(61 \%)$ followed by a SAH (59\%), an CC $(56 \%)$, and an EDH (16\%). Sixty-nine patients (64\%) presented with a skull fracture. Many patients $(69 \%)$ sustained a combination of t-ICH. The most frequent combinations were $\mathrm{ASDH}+\mathrm{CC}+\mathrm{SAH}(17 \%)$ and $\mathrm{ASDH}+\mathrm{SAH}(16 \%)$.

Twenty-eight patients underwent a total of 38 surgical interventions. The most frequently performed procedure was a decompressive craniotomy (29\%) followed by a craniotomy with evacuation of the hemorrhage (28\%), placement of an intracranial pressure measurement device (24\%), and placement of an external ventricular drain (21\%).

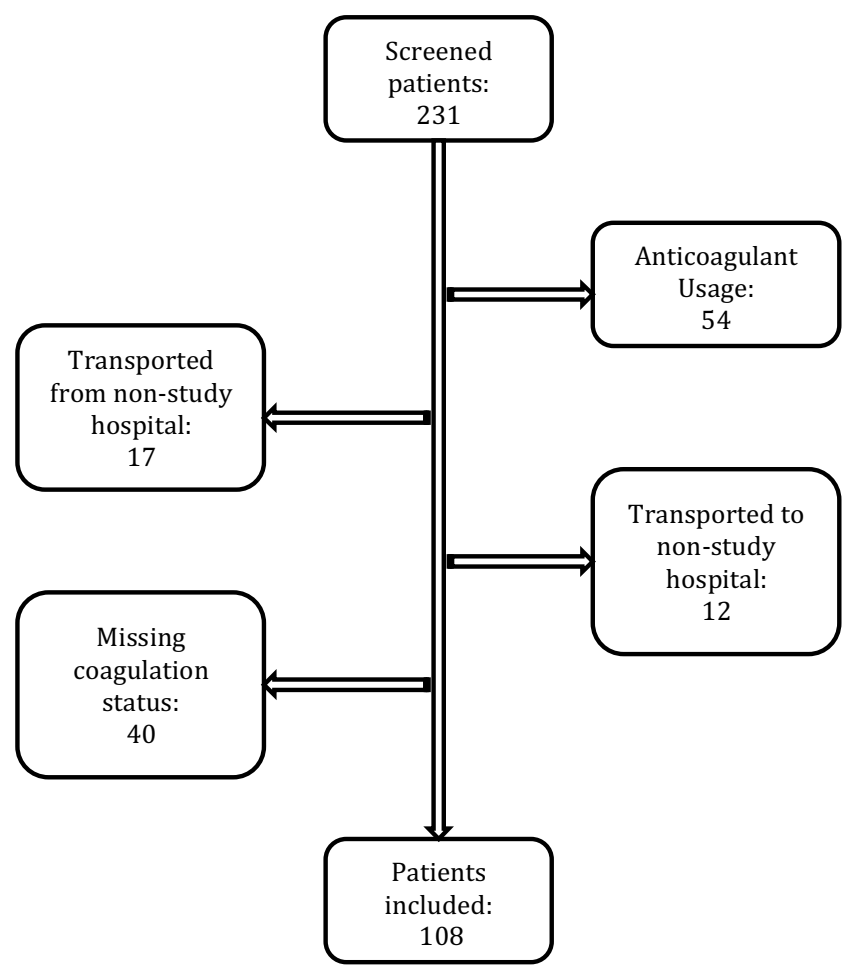

Fig. 1 Flowchart

\section{Coagulopathy at ED presentation vs. no coagulopathy}

Of the 108 patients, thirteen patients (12\%) showed a coagulopathy on ED presentation leaving 95 patients $(88 \%)$ with undisturbed coagulation parameters at presentation to the ED. Baseline parameters, including age and AIS at arrival, were similar in the coagulopathy and no-coagulopathy groups (Table 2). Male preponderance was existent in the coagulopathy group compared to the no-coagulopathy group (77 vs. $47 \%$ ). Patients in the coagulopathy group presented with a lower GCS (median (IQR) GCS of 5 (5) compared to 13 (6) in the no-coagulopathy group $(p=0.00)$ ), which reflects the severity of the injury. The majority of patients in the coagulopathy and no-coagulopathy group had an ASA score of $\leq 2$ before trauma ( $98 \%$ and $92 \%$, respectively), which reflects the healthy status of patients in both groups. In the coagulopathy group, most patients sustained a combination of ASDH+CC+ SAH (46\%) followed by ASDH+SAH (23\%) and the third most common intracranial hemorrhage was an ASDH $(15 \%)$. In the no-coagulopathy group, most patients sustained a combination of ASDH+SAH (16\%) followed by ASDH+ $\mathrm{CC}+\mathrm{SAH}(13 \%)$ and $\mathrm{ASDH}+\mathrm{CC}(12 \%)$.

The proportion of patients that required surgery was twice as large in the coagulopathy group relative to the no-coagulopathy group ( $46 \%$ vs. $23 \%$ ) with an OR of 2.8 (95\% CI $0.87-9.35$ ), and patients in the coagulopathy group were more likely to be admitted to the ICU than patients in the no-coagulopathy group (92\% vs. 62\%) with an OR of 7.3 (95\% CI 0.91-58.71). 
Table 1 Clinical characteristics of the study cohort, consisting of 108 patients who sustained an acute traumatic intracranial bleeding. Continuous variables denoted as means with standard deviation (SD), categorical data as counts (\%). Median was used when there was nonnormal distribution with interquartile range (IQR)

\begin{tabular}{ll}
\hline Characteristics & $n(\%)$ \\
\hline Total patients & $108(100)$ \\
Age, median (IQR) & $60(31)$ \\
Male sex & $55(51)$ \\
ASA score & \\
Normal healthy person & $67(62)$ \\
Person with a mild systemic disease & $38(35)$ \\
Patient with a severe systemic disease & $3(3)$ \\
GCS on admission, median (IQR) & $12(8)$ \\
Type of lesion * & \\
ASDH & $66(61)$ \\
EDH & $17(16)$ \\
CC & $60(56)$ \\
SAH & $64(59)$ \\
Combination of hemorrhages & $75(69)$ \\
Extracranial AIS on admission, median (IQR) & $2(3)$ \\
Number of CT-scans performed, median (IQR) & $2(1)$ \\
Midline shift & $31(29)$ \\
Skull fracture & $69(64)$ \\
Progression of hemorrhagic injury & $38(35)$ \\
In-hospital mortality & $22(20)$ \\
Coagulation Parameters & \\
HB, mean (SD) & $8.2(1.1)$ \\
Platelets, mean (SD) & $230.7(67.7)$ \\
Hypertension & $24(22.2)$ \\
Duration of hospital stay, median (IQR) & $8(11)$ \\
Admitted to ICU & $71(66)$ \\
Patients operated & 28 \\
Operations performed & 38 \\
Evacuation of hemorrhage & $10(10)$ \\
Intracranial pressure monitoring device & $9(8)$ \\
Decompressive craniectomy & $11(10)$ \\
External ventricular drain & \\
\hline Sol & \\
\hline
\end{tabular}

Some patients had multiple intracranial lesions

ASA American Society of Anesthesiologists, GCS Glasgow Coma Scale, $A S D H$ acute subdural hemorrhage, $E D H$ epidural hemorrhage, $C C$ cerebral contusion, $S A H$ subarachnoid hemorrhage, AIS Abbreviated Injury Scale, CT scan computed tomography scan, $H B$ hemoglobin, ICU intensive care unit

The median number of CT scans taken during admission was similar for both groups being two per patient. There was a higher proportion of skull fractures in the coagulopathy group than in the no-coagulopathy group (77\% vs. $62 \%)$ with an OR of 2.0 (95\% CI $0.53-7.87$ ). Patients with coagulopathy presented with a higher rate $(62 \%)$ of midline shift compared to the no-coagulopathy group (62 vs. $24 \%$ ) with an OR of 5.0
(1.49-16.83). Overall, a total of 22 patients (20\%) died. CT scan analysis demonstrated that a total of 38 patients (35\%) suffered from PHI. A higher proportion of PHI was found in the coagulopathy group (54\%) compared to the nocoagulopathy group (33\%) with an OR of 2.4 (95\% CI 0.8 8.0). Although the former was non-significant, the observation that a higher proportion of patients died in the coagulopathy group (77\%) compared to the no-coagulopathy group (13\%) with an OR 23.06 (95\% CI 5.5-95.9)) was significant.

In the subgroup of patients with only focal lesions, thirteen (13\%) showed a coagulopathy on ED presentation of 99 patients. The relation with future events was almost similar: PHI OR 2.3 (95\% CI 0.7-7.3), for surgery OR 2.5 (95\% CI 0.7 8.1), admission to the ICU OR 6.4 (95\% CI 0.8-52), and mortality OR 20.6 (95\% CI 4.9-86).

\section{Coagulopathy parameters}

Patients with coagulopathy at ED presentation had a mean INR of 1.19 (95\% CI 1.12-1.26), a mean APTT of 38.7 $(32.0-45.3) \mathrm{s}$ and a platelet count of $170(93-247) \times 10^{3} / \mu \mathrm{l}$. Patients without a coagulopathy had a mean INR of 1.00 (1.00-1.02), APTT of $27.0(26.4-27.6) \mathrm{s}$, and a platelet count of $240(229-250) \times 10^{3} / \mu 1$. In the coagulopathy group, $53.4 \%$ demonstrated an elevated APTT, $46.2 \%$ had a low platelet count, and an INR $>1.2$ of was observed in $15.4 \%$ of patients (Table 2). A medical history of hypertension (HT) was found in 24 patients, 23 in the no-coagulopathy group, and one in the coagulopathy group ( $p$ value 0.15 ). Patients with HT used diuretics $(50 \%)$, beta blockers $(33 \%)$, angiotensinconverting-enzyme inhibitors (21\%), angiotensin-II-receptor blockers $(13 \%)$, calcium channel blockers $(8 \%)$, or no medication $(8 \%)$.

\section{Time course of coagulopathy and association with outcome}

Patients in the studied cohort often developed a coagulopathy in the days after trauma. Within $96 \mathrm{~h}$ after trauma, a total of 29 (27\%) patients developed a new coagulopathy. This study found an incidence of coagulopathy of $12.0 \%, 7.9 \%, 11.5 \%$, and $0 \%$ for 0 to $24 \mathrm{~h}, 24$ to $48 \mathrm{~h}, 48$ to $72 \mathrm{~h}$, and 72 to $96 \mathrm{~h}$ after hospital admission respectively (Fig. 2). In fourteen patients, the coagulopathy resolved within $96 \mathrm{~h}$ after trauma.

Six patients (three coagulopathic and three noncoagulopathic) died the first $24 \mathrm{~h}$ after admission, while four patients (two coagulopathic and two non-coagulopathic) died between 24 and $48 \mathrm{~h}$ following hospital admission. Furthermore, two patients (both coagulopathic) died between 48 and $72 \mathrm{~h}$ and one patient (non-coagulopathic) died between 72 and $96 \mathrm{~h}$ after admission. An additional nine patients (six coagulopathic and three non-coagulopathic) died $96 \mathrm{~h}$ after 
Table 2 Characteristics of nocoagulopathy and coagulopathy group. Continuous variables denoted as means with standard deviation (SD), or medians with interquartile range (IQR) and categorical data as proportions $(\%)$

\begin{tabular}{|c|c|c|c|}
\hline Characteristics & $\begin{array}{l}\text { Non-coagulopathic } \\
\text { patients, } n(\%)\end{array}$ & $\begin{array}{l}\text { Coagulopathic patients at } \\
\text { admission, } n(\%)\end{array}$ & $p$ value \\
\hline Total patients & $95(88)$ & $13(12)$ & \\
\hline Age, median (IQR) & $61(31)$ & $58(35)$ & 0.92 \\
\hline Male sex & $45(47)$ & $10(77)$ & 0.05 \\
\hline \multicolumn{4}{|l|}{ ASA score } \\
\hline A normal healthy person & $62(65)$ & $5(39)$ & 0.06 \\
\hline Patient with a mild systemic disease & $31(33)$ & $7(53)$ & 0.29 \\
\hline Patient with a severe systemic disease & $2(2)$ & $1(8)$ & 0.25 \\
\hline GCS on admission, median (IQR) & $13(6)$ & $5(5)$ & 0.00 \\
\hline \multicolumn{4}{|l|}{ Type of lesion* } \\
\hline ASDH & $55(58)$ & $11(85)$ & 0.07 \\
\hline EDH & $17(18)$ & $0(0)$ & 0.10 \\
\hline $\mathrm{CC}$ & $52(55)$ & $8(62)$ & 0.65 \\
\hline $\mathrm{SAH}$ & $54(57)$ & $10(77)$ & 0.17 \\
\hline Combination of hemorrhage & $64(67)$ & $11(85)$ & 0.21 \\
\hline Extracranial AIS on admission, median (IQR) & $2(3)$ & $0(2)$ & 0.14 \\
\hline CT-scans performed, median (IQR) & $2(1)$ & $2(2)$ & 0.55 \\
\hline Midline shift & $23(24)$ & $8(62)$ & 0.01 \\
\hline Skull fracture & $59(62)$ & $10(77)$ & 0.30 \\
\hline Progression of hemorrhagic injury & $31(33)$ & $7(54)$ & 0.10 \\
\hline In-hospital mortality & $12(13)$ & $10(77)$ & 0.00 \\
\hline $\mathrm{HB}$, mean (SD) & $8.3(0.9)$ & $7.3(1.9)$ & 0.00 \\
\hline Platelets, mean (SD) & $239(49.9)$ & $170(127.5)$ & 0.00 \\
\hline Hypertension & $23(24.2)$ & $1(7.7)$ & 0.15 \\
\hline Duration of hospital stay, median (IQR) & $8(9)$ & $3(14)$ & 0.52 \\
\hline Admitted to ICU & $59(62)$ & $12(92)$ & 0.03 \\
\hline Patients operated & $22(23)$ & $6(43)$ & 0.16 \\
\hline Operation performed & $29(31)$ & $8(62)$ & 0.08 \\
\hline Evacuation of hemorrhage & $8(8)$ & $2(15)$ & 0.42 \\
\hline Intracranial pressure monitoring device & $7(6)$ & $3(23)$ & 0.10 \\
\hline Decompressive craniectomy & $7(6)$ & $3(23)$ & 0.10 \\
\hline External ventricular drain & $8(8)$ & $0(0)$ & 0.28 \\
\hline
\end{tabular}

Coagulopathy was defined as an international normalized ratio (INR) $>1.2$ and/or a platelet count below $150 \times$ $10^{3} / \mu 1$ and/or activated partial thromboplastin time (APTT) $>34.5 \mathrm{~s}$

ASA American Society of Anesthesiologists, GCS Glasgow Coma Scale, ED emergency department, ASDH acute subdural hemorrhage, $E D H$ epidural hemorrhage, $C C$ Cerebral Contusion, $S A H$ subarachnoid hemorrhage, AIS Abbreviated Injury Scale, CT scan computed tomography scan, $H B$ hemoglobin, $I C U$ intensive care unit, ICP intracranial pressure hospital admission. A total of 22 patients died of which ten were coagulopathic upon presentation to the ED (Fig. 3).

\section{Discussion}

In this observational cohort study of 108 patients with an acute t-ICH, thirteen patients ( $12 \%$ ) developed a coagulopathy within $24 \mathrm{~h}$ after trauma and $22(20 \%)$ patients died within $96 \mathrm{~h}$ after admission. The new coagulopathy was not confined to the first $24 \mathrm{~h}$ and even occurred at $72 \mathrm{~h}$ after trauma.
Furthermore, coagulopathy was associated with PHI, surgical intervention, and strongly associated with in-hospital death. Patients who sustained a TBI should therefore be closely monitored because they can develop a coagulopathy and consequently deteriorate in the days following trauma. Although coagulation derailments have been studied previously in the general TBI population, this is one of the first reports showing the occurrence of a coagulopathy in patients who sustained tICH $[2,10]$. In a recent review by Epstein and colleagues, 22 retrospective and prospective cohort studies were analyzed to examine the incidence and clinical outcome of coagulopathy 
Fig. 2 Time course of coagulopathy. $Y$-axis, total patients. The incidence of coagulopathy was $12.0 \%, 7.9 \%$, $11.5 \%$, and $0 \%$ for 0 to $24 \mathrm{~h}, 24$ to $48 \mathrm{~h}, 48$ to $72 \mathrm{~h}$, and 72 to $96 \mathrm{~h}$ after admission, respectively (defined as an international normalized ratio $(\mathrm{INR})>1.2 \mathrm{and} /$ or a platelet count below $150 \times$ $10^{3} / \mu 1$ and/or activated partial thromboplastin time (APTT) > $34.5 \mathrm{~s})$

\section{Timecourse coagulopathy}

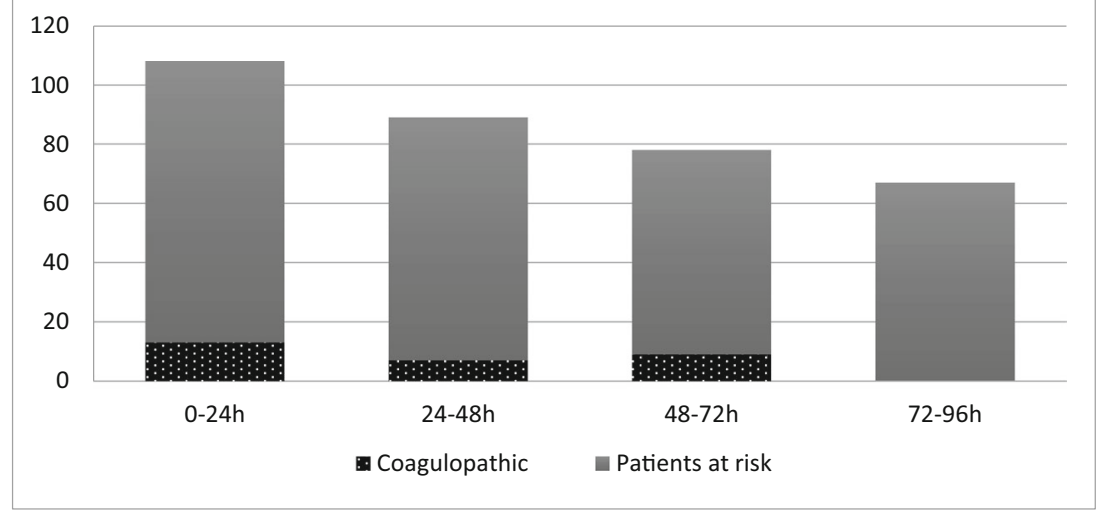

in the general TBI population [8]. They found a pooled incidence of $35.2 \%$ (95\% CI 29.0-41.4), which is higher than reported in this study (27\%). However, the definition of coagulopathy as reported by this review was not consistent and included different combinations of INR, platelet count, prothrombin time (PT), APTT, fibrinogen, disseminated intravascular coagulation score, modified coagulopathy score, and alpha-2 plasmin inhibitor value. In general, the incidence of coagulopathy after TBI appears to be highly dependable on the used cutoff value and time of blood draw. The current study made use of the INR as a marker for coagulopathy as opposed to a prolonged PT, to avoid interassay variation in PT analyses used by different institutions.

There remains a discrepancy in reports on the time course of coagulopathy. Some studies report an increase of TBI patients who develop a coagulopathy after the presentation at the $\mathrm{ED}$, others report a decrease in coagulopathy incidence $[3,15$, $16,22,30,32]$. This study found an incidence of around $10 \%$ for 24,48 , and $72 \mathrm{~h}$ after trauma, indicating that patients with TBI remain at risk for developing a coagulopathy days after the trauma. Only after $72 \mathrm{~h}$, no patients developed a new coagulopathy in this cohort.

This study found a higher proportion of PHI in the coagulopathy group than in the no-coagulopathy group (54\% vs. $33 \%$ ) (OR of 2.4 (95\% CI 0.8-8.0)) which is comparable to other studies $[1,18]$. Despite the heterogenic definition and cutoff values used, the majority of studies have linked coagulopathy with an increased risk of PHI $[1,7,18,20,26]$, although some studies report no relation between PHI and coagulopathy (i.e., unchanged additional CT scans in patients with coagulopathy after TBI) $[19,29]$. Naturally, part of the $\mathrm{PHI}$ is explained by the severity of the primary brain injury more so than by secondary PHI, induced by (unrecognized) coagulopathy.

Finally, this study found a strong relation between coagulopathy and in-hospital mortality. This is similar to recent studies that reported a much higher mortality rate for coagulopathic patients than for patients who did not have a coagulopathy. Moreover, in these studies, the same cutoff value was used to define a coagulopathy (INR > 1.2) $[1,32]$.
Fig. 3 Mortality in cohort. A total of 22 patients died during admission of which ten $45 \%$ (odds ratio $23.06(95 \%$ confidence interval 5.5-95.9)) were coagulopathic at presentation to the emergency department

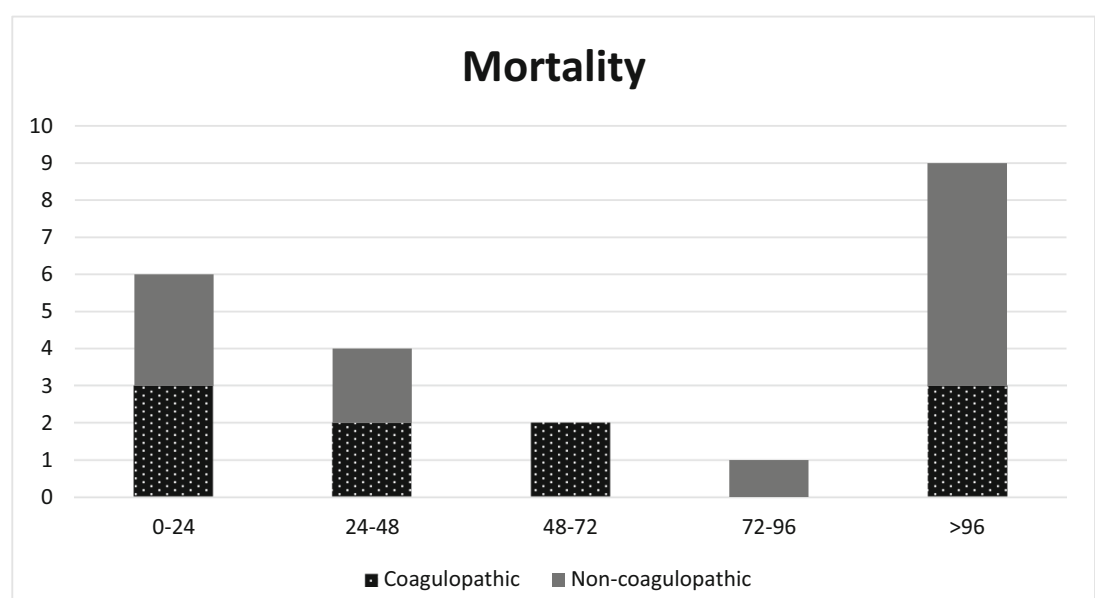




\section{Strengths and limitations}

Most studies that observed a correlation between coagulopathy and TBI focused on the broad TBI population or on specific subgroups based on GSC, time interval, age, or the presence of extracranial injury. This is one of the first studies that identify a coagulopathy in patients with intracranial hemorrhages after TBI. In patients with an intracranial hemorrhage, proper hemostasis is vital to prevent the initial hemorrhage to further expand and cause secondary injury.

Selection bias is an important consideration in this observational study. In the current cohort, the coagulation assessment (INR, APTT, and platelet analysis) was performed following indication by the treating physician, which explains why less information was available on coagulopathy status in the days after presentation. A variation in iatrogenic correction of coagulopathy could also interfere with the time course of coagulopathy. This study did not take correction of coagulation into account. Because it was not in the protocol to have the coagulation status checked the days after trauma, the relationship between clinical outcome (mortality, surgical intervention and PHI) and coagulation status has only been explored for blood drawn at ED presentation.

In the participating hospitals, performing an additional CT scan occurs on indication only. This may lead to biased PHI rates, as neurologically unstable patients are usually indicated for an additional CT scan. Patients with t-ICH who remained clinically stable did not have a follow-up CT scan.

Patients with a coagulopathy within $24 \mathrm{~h}$ after trauma presented with a lower mean GCS than patients without a coagulopathy (6.1 vs. 10.9) and thus have a worse initial prognosis. Whether or not the coagulopathy is an independent risk factor and/or possibly (partly) causes intracranial bleeding are topics for future research.

\section{Future}

An important question is whether an intervention exists that can reduce PHI, induced by the coagulopathy after TBI. To tackle this clinical problem, one must find a therapeutic target, constructed from pathophysiological insights. However, coagulopathy after TBI is the result of a complex mechanism that is still largely unknown [25]. Due to the lack of understanding on how TBI induces coagulopathy, no evidence-based clinical intervention is available to prevent or treat the contribution of coagulopathy to the development of secondary injury after TBI.

In the future, the authors will set up a multicenter casecohort study within the already active Net-QuRe and CENTER-TBI studies to identify differences in specific coagulation parameters between patients with $\mathrm{PHI}$ and patients who did not develop secondary hemorrhages [24].

\section{Conclusion}

Coagulopathy after TBI is a complex mechanism that is not fully understood. Patients who sustained an intracranial hemorrhagic injury remained at risk for developing a coagulopathy until $72 \mathrm{~h}$ after trauma. Early coagulopathy is associated with PHI, surgical intervention, and increased in-hospital mortality of patients who sustained t-ICH. This indicates that more extensive etiological research with a detailed analysis of the coagulation status after TBI is needed to improve critical care, lower mortality, and improve neurological prognosis for these patients.

Funding This work was supported by The European Union seventh Framework Program (grant 602150) for Collaborative European NeuroTrauma Effectiveness Research in Traumatic Brain Injury (CENTER-TBI) and Brain Foundation of The Netherlands (Hersenstichting Nederland) for Neurotraumatology Quality Registry (Net-QuRe).

\section{Compliance with ethical standards}

Conflict of interest The authors declare that they have no conflict of interest.

Ethical approval For this type of study, formal consent is not required.

Open Access This article is distributed under the terms of the Creative Commons Attribution 4.0 International License (http:// creativecommons.org/licenses/by/4.0/), which permits unrestricted use, distribution, and reproduction in any medium, provided you give appropriate credit to the original author(s) and the source, provide a link to the Creative Commons license, and indicate if changes were made.

\section{References}

1. Allard CB, Scarpelini S, Rhind SG, Baker AJ, Shek PN, Tien H, Fernando M, Tremblay L, Morrison LJ, Pinto R, Rizoli SB (2009) Abnormal coagulation tests are associated with progression of traumatic intracranial hemorrhage. J Trauma 67(5):959-967

2. Bershad EM, Farhadi S, Suri MFK, Feen ES, Hernandez OH, Selman WR, Suarez JI (2008) Coagulopathy and inhospital deaths in patients with acute subdural hematoma. J Neurosurg 109:664669

3. Carrick MM, Tyroch AH, Youens CA, Handley T (2005) Subsequent development of thrombocytopenia and coagulopathy in moderate and severe head injury: support for serial laboratory examination. J Trauma 58:725-729 discussion 729-30

4. Chang R, Cardenas JC, Wade CE, Holcomb JB (2016) Advances in the understanding of trauma-induced coagulopathy. Blood 128: 1043-1050

5. Cohen MJ, Brohi K, Ganter MT, Manley GT, Mackersie RC, Pittet J-F (2007) Early coagulopathy after traumatic brain injury: the role of hypoperfusion and the protein C pathway. J Trauma 63:1254 1261 discussion 1261-1262

6. Daabiss M (2011) American Society of Anaesthesiologists physical status classification. Indian J Anaesth 55(2):111-115 
7. DeOliveira Manoel AL, Neto AC, Veigas PV, Rizoli S (2015) Traumatic brain injury associated coagulopathy. Neurocrit Care 22:34-44

8. Epstein DS, Mitra B, O'Reilly G, Rosenfeld JV, Cameron PA (2014) Acute traumatic coagulopathy in the setting of isolated traumatic brain injury: a systematic review and meta-analysis. Injury 45:819-824

9. Finfer SR, Cohen J (2001) Severe traumatic brain injury. Resuscitation 48:77-90

10. Folkerson LE, Sloan D, Cotton BA, Holcomb JB, Tomasek JS, Wade CE (2015) Predicting progressive hemorrhagic injury from isolated traumatic brain injury and coagulation. Surgery 158:655661

11. Franschman G, Boer C, Andriessen TMJC, van der Naalt J, Horn J, Haitsma I, Jacobs B, Vos PE (2012) Multicenter evaluation of the course of coagulopathy in patients with isolated traumatic brain injury: relation to CT characteristics and outcome. J Neurotrauma 29:128-136

12. Ghajar J (2000) Traumatic brain injury. Lancet 356:923-929

13. Giesen PLA, Nemerson Y (2000) Tissue factor on the loose. Semin Thromb Hemost 26:379-384

14. Giesen PLA, Rauch U, Bohrmann B, Kling D, Roqué M, Fallon JT, Badimon JJ, Himber J, Riederer MA, Nemerson Y (1999) Bloodborne tissue factor: another view of thrombosis. Proc Natl Acad Sci 96:2311-2315

15. Greuters S, Van Den Berg A, Franschman G, Viersen VA, Beishuizen A, Peerdeman SM, Boer C, ALARM-BLEEDING investigators (2011) Acute and delayed mild coagulopathy are related to outcome in patients with isolated traumatic brain injury. Crit Care 15:R2

16. Halpern CH, Reilly PM, Turtz AR, Stein SC (2008) Traumatic coagulopathy: the effect of brain injury. J Neurotrauma 25:9971001

17. Harhangi BS, Kompanje EJO, Leebeek FWG, Maas a IR (2008) Coagulation disorders after traumatic brain injury. Acta Neurochir 150:165-175 discussion 175

18. Juratli TA, Zang B, Litz RJ, Sitoci K-H, Aschenbrenner U, Gottschlich B, Daubner D, Schackert G, Sobottka SB (2014) Early hemorrhagic progression of traumatic brain contusions: frequency, correlation with coagulation disorders, and patient outcome: a prospective study. J Neurotrauma 31:1521-1527

19. Kaups KL, Davis JW, Parks SN (2004) Routinely repeated computed tomography after blunt head trauma: does it benefit patients? J Trauma 56:475-480 discussion 480-1

20. Kurland D, Hong C, Aarabi B, Gerzanich V, Simard JM (2012) Hemorrhagic progression of a contusion after traumatic brain injury: a review. J Neurotrauma 29:19-31

21. Laroche M, Kutcher ME, Huang MC, Cohen MJ, Manley GT (2012) Coagulopathy after traumatic brain injury. Neurosurgery 70:1334-1345

22. Lustenberger T, Talving P, Kobayashi L, Barmparas G, Inaba K, Lam L, Branco BC, Demetriades D (2010) Early coagulopathy after isolated severe traumatic brain injury: relationship with hypoperfusion challenged. J Trauma 69:1410-1414

23. Maas AIR, Stocchetti N, Bullock R (2008) Moderate and severe traumatic brain injury in adults. Lancet Neurol 7:728-741
24. Maas AIR, Menon DK, Steyerberg EW, CENTER-TBI investigators (2015) Collaborative European neurotrauma effectiveness research in traumatic brain injury (CENTER-TBI): A prospective longitudinal observational study. Neurosurgery 76:67-80

25. Maegele M (2013) Coagulopathy after traumatic brain injury: Incidence, pathogenesis, and treatment options. Transfusion. 53: 28S-37S

26. Oertel M, Kelly DF, McArthur D, Boscardin WJ, Glenn TC, Lee JH, Gravori T, Obukhov D, McBride DQ, Martin NA (2002) Progressive hemorrhage after head trauma: predictors and consequences of the evolving injury. J Neurosurg 96:109-116

27. Rezaie AR (2001) Vitronectin functions as a cofactor for rapid inhibition of activated protein $\mathrm{C}$ by plasminogen activator inhibitor-1: implications for the mechanism of profibrinolytic action of activated protein C. J Biol Chem 276:15567-15570

28. Roozenbeek B, Maas AIR, Menon DK (2013) Changing patterns in the epidemiology of traumatic brain injury. Nat Rev Neurol 9:231236

29. Stein SC, Spettell C, Young G, Ross SE (1993) Delayed and progressive brain injury in closed-head trauma: radiological demonstration. Neurosurgery 32:25-30 discussion 30-1

30. Stein DM, Dutton RP, Kramer ME, Scalea TM (2009) Reversal of coagulopathy in critically ill patients with traumatic brain injury: recombinant factor VIIa is more cost-effective than plasma. $\mathrm{J}$ Trauma 66:63-72 discussion 73-75

31. Talving P, Lustenberger T, Lam L, Inaba K, Mohseni S, Plurad D, Green DJ, Demetriades D (2011) Coagulopathy after isolated severe traumatic brain injury in children. J Trauma 71:1205-1210

32. Wafaisade A, Lefering R, Tjardes T, Wutzler S, Simanski C, Paffrath T, Fischer P, Bouillon B, Maegele M (2010) Acute coagulopathy in isolated blunt traumatic brain injury. Neurocrit Care 12: 211-219

Comments Traumatic brain injury aggravated by concomitant coagulopathy is common and associated with increased rates of mortality, morbidity and disability. When coagulopathy is present, rapid reversal should be a high priority to prevent hemorrhage expansion leading to further secondary both intracranial and extracranial trauma consequences. Even though the topic has been discussed in several previous publications, the authors contributed interesting new data that invite further investigations. For instance, antifibrinolytics that have been studied in major trauma to prevent excessive blood loss such as tranexamic acid given early might be promising therapeutic candidates. The authors are congratulated for another valuable contribution to our neurosurgical and neurotraumatological knowledge, despite the study shortcomings, adequately and critically discussed within the manuscript.

Markus F. Oertel

Zurich, Switzerland

Publisher's note Springer Nature remains neutral with regard to jurisdictional claims in published maps and institutional affiliations. 